\title{
The Use of Kalman Filtering and Correlation Techniques in Analytical Calibration Procedures
}

\author{
H. C. Smit \\ University of Amsterdam, Nieuwe Achtergracht 166, 1018 WV Amsterdam, The Netherlands
}

Accepted: June 24, 1985

Different chemometric methods to improve calibrations are described. A Kalman filter is applied for processing and predicting slowly varying parameters of a linear calibration graph. The results are used for the evaluation of unknown samples, and for deciding whether to calibrate again or to analyze the next unknown sample. Another approach of the calibration problem, particularly in chromatography, is the use of correlation techniques. The noise reduction property of correlation chromatography is used to extend the calibration graph to very low concentrations. Furthermore, an experimental technique to determine a calibration curve and the unknown sample simultaneously under exactly the same conditions is described.

Key words: calibration; chromatography; correlation techniques; Kalman filter.

\section{Introduction}

The computer has added a new dimension to analytical chemistry. Chemometrics, the application of mathematical and statistical techniques, is improving the quality of the analytical results concerning accuracy, precision, time, and costs, and has created new possibilities. An extended number of chemometric procedures are now available and are being increasingly applied in practice.

However, the "off-line" application of chemometric procedures, i.e., the processing of data or signals already obtained with common analytical methods like titration, chromatography, spectroscopy, etc., is dominating. The computer is generally used as an off-line calculation machine. The incorporation of the computer into existing analytical methods or the development of new meth-

About the Author, Paper: H. C. Smit is with the University of Amsterdam's Laboratory for Analytical Chemistry. The work he describes was supported in part by the Netherlands Research Organization ZWO. ods based on the capabilities of the computer is not far developed; the intelligent analyzer is still in its infancy.

In this paper, some examples of chemometric on-line computer applications in an analytical procedure and analytical method are given. The calibration, which is very important in analytical chemistry, is emphasized in both examples-a generally applicable procedure using an optimum recursive parameter estimation technique (vector Kalman filter), and a method developed for one particular analytical technique (chromatography). More details concerning the basic theory are given in $[1]^{1}$.

\section{Calibration and Optimum Estimation}

An analytical system is usually very complex and includes chemical, optical, electrical, and mechanical parts. All these parts are subject to several influences, like contamination, changes in temperature, humidity, etc., and, of course, aging. These influences result in a decrease of the quality of the analytical data. Two main

\footnotetext{
${ }^{1}$ Figures in brackets indicate literature references.
} 
components can be distinguished: a stochastic component resulting in stationary random fluctuations, and a semi-random component caused by irreversible processes like the mentioned contamination, aging etc. This semi-random component has a non-stationary nature and a regular calibration is required to maintain the quality of the results respectively to reduce the influence of the drift in the calibration parameters. Ignored drift seriously affects the accuracy in the analytical results and various off-line drift correction procedures have been proposed [2-6].

A Kalman filter enables on-line drift compensation, particularly suitable in the case of automated analytical procedures. An optimum recursive estimator like a Kalman filter requires a model of both the system or the signal process, including the system noise and the measurement (observation) noise. Using an appropriate model, the Kalman filter can predict (estimate) future values of the changing parameters and the samples can be evaluated using these predicted parameters. The estimation may also be used to determine when a recalibration is required. The criterion is a given preset precision of the results. The final goal is to analyze samples with a predetermined minimum accuracy.

\section{System Model}

A state space model is used to describe the system. The linear discrete dynamic system

$$
\begin{aligned}
& \mathbf{x}(k)=\mathbf{F}(k) \mathbf{x}(k-1)+\mathbf{w}(k-1) \\
& z(k)=\mathbf{h}^{\prime}(k) \mathbf{x}(k)+v(k)
\end{aligned}
$$

where

$$
\begin{array}{ll}
k & : a \text { time or a sequence number } \\
\mathbf{x}(k) & : n \times 1 \text { state vector } \\
\mathbf{F}(k) & : n \times n \text { transition matrix } \\
\mathbf{h}^{\prime}(k) & : 1 \times n \text { measurement vector } \\
z(k) \quad: \text { measured signal } \\
\mathbf{w}(k-1): n \times 1 \text { system noise vector } \\
v(k) \quad: \text { scalar measurement noise }
\end{array}
$$

is representative for many analytical systems (fig. 1). The model is tinear because neither in the transition matrix $\mathbf{F}(k)$ nor in the measurement vector $\mathbf{h}^{r}(k)$ parameters $x(k)$ are present. A commonly used calibration graph is

$$
y=a \cdot c+b
$$

where

$a:$ sensitivity
$b:$ intercept $c$ : concentration

$y$ : measurement

Reformulation gives

$$
y=(c, 1)\left(\begin{array}{l}
a \\
b
\end{array}\right)
$$

Using

$$
\begin{aligned}
& \mathbf{x}=\left(\begin{array}{l}
a \\
b
\end{array}\right) \quad \mathbf{F}=\left(\begin{array}{ll}
1 & 0 \\
0 & 1
\end{array}\right) \\
& \mathbf{h}^{\prime}=(c, 1) \text { and } z=y
\end{aligned}
$$

and adding system noise and measurement noise results in a dynamic calibration curve

$$
\begin{aligned}
& \left(\begin{array}{l}
a(k) \\
b(k)
\end{array}\right)=\left(\begin{array}{ll}
1 & 0 \\
0 & 1
\end{array}\right)\left(\begin{array}{l}
a(k-1) \\
b(k-1)
\end{array}\right)+\left(\begin{array}{l}
w_{1}(\mathrm{k}-1) \\
w_{2}(k-1)
\end{array}\right) \\
& z(k)=(c, 1) \cdot\left(\begin{array}{l}
a(k) \\
b(k)
\end{array}\right)+v(k) .
\end{aligned}
$$

A common situation in practice is that the parameters of the calibration curve are slowly varying in time. Stochastic variations can be represented by the system noise, introduced in the model. However, in practice often a deterministic variation of the calibration parameter can be observed; particularly an extension of the model with a linear drift increases the usability.

A single parameter (state) $x$, affected by a linear drift in the sequence $k$ can be written as

$$
x(k)=d k+e
$$

where

$$
\begin{aligned}
& d: \text { (constant) drift parameter } \\
& e: x(0)
\end{aligned}
$$

Evaluation of eq (8) leads to

$$
\begin{aligned}
x(k)= & d(k-1)+d+e \\
& =x(k-1)+d .
\end{aligned}
$$

The introduction of system noise and measurement noise gives

$$
\begin{aligned}
& \left(\begin{array}{l}
x(k) \\
d(k)
\end{array}\right)=\left(\begin{array}{ll}
1 & 1 \\
0 & 1
\end{array}\right)\left(\begin{array}{l}
x(k-1) \\
d(k-1)
\end{array}\right)+\left(\begin{array}{l}
w_{1}(k-1) \\
w_{2}(k-1)
\end{array}\right) \\
& z(k)=(1,0)\left(\begin{array}{l}
x(k) \\
d(k)
\end{array}\right)+v(k) .
\end{aligned}
$$


Figure 1-Linear dynamic system.

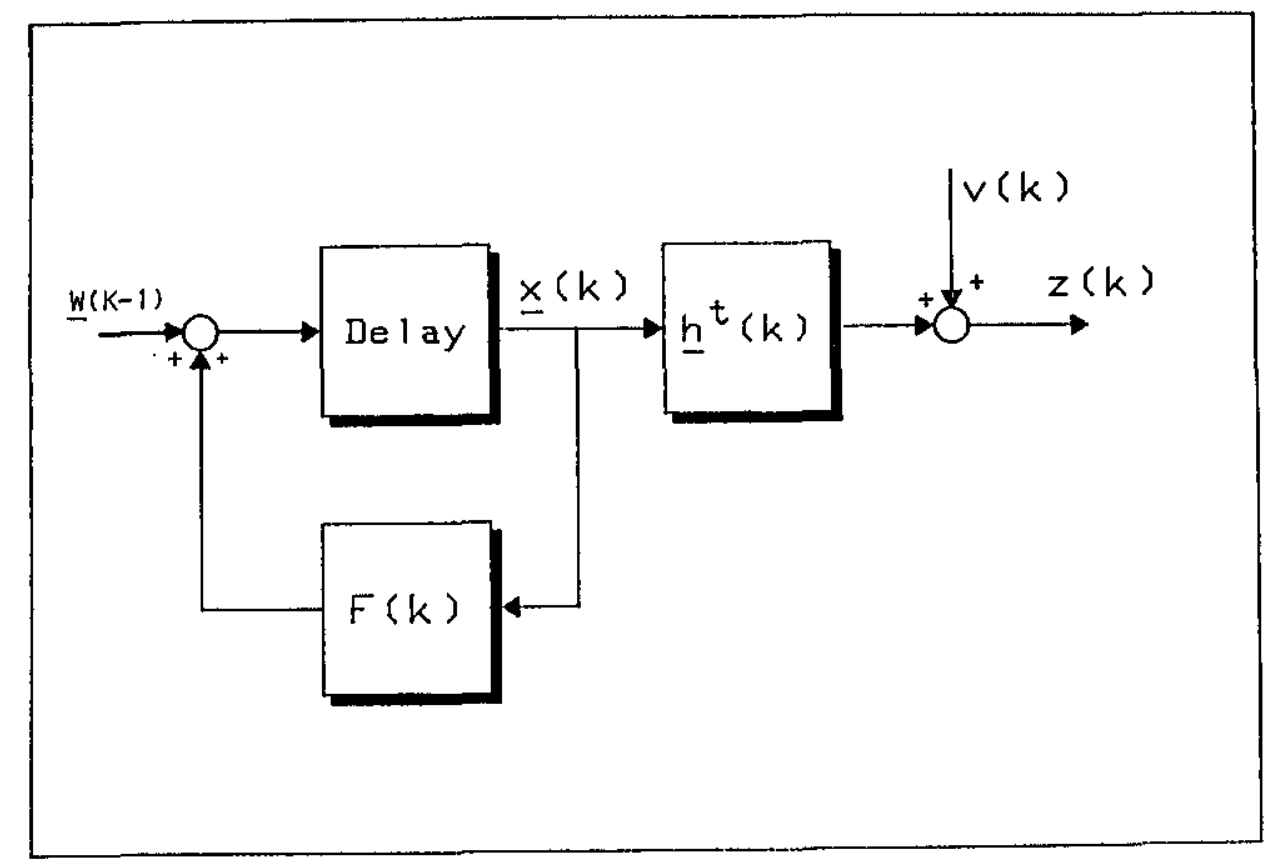

If both the sensitivity $a$ and the intercept $b$ are influenced by random drift, two extra parameters have to be introduced in the original model. The final model is

$$
\begin{aligned}
& \begin{array}{l}
a(k) \\
b(k) \\
a(k) \\
\beta(k)
\end{array}=\begin{array}{ll}
1010 & a(k-1) \\
0101 & b(k-1) \\
0010 & \alpha(\mathrm{k}-1) \\
0001 & \beta(k-1)
\end{array}+\begin{array}{l}
w_{1}(k-1) \\
w_{2}(k-1) \\
w_{3}(k-1) \\
w_{4}(k-1)
\end{array} \\
& z(k)=(c, 1,0,0) \quad \begin{array}{l}
a(k) \\
b(k) \\
\alpha(k) \\
\beta(k)
\end{array} \quad+v(k) .
\end{aligned}
$$

The observability matrix is

$$
M=\begin{array}{llll}
c_{1} & c_{2} & c_{3} & c_{4} \\
1 & 1 & 1 & 1 \\
0 & c_{2} & 2 c_{3} & 3 c_{4} \\
0 & 1 & 2 & 3
\end{array}
$$

The system is observable if there are at least two different concentrations.

\section{Sample Evaluation and Recalibration}

Assuming the transition matrix $\mathbf{F}(k)$ in eq (11) is known exactly and the measurement vector $\mathbf{h}^{t}(k)$ is known from the calibration, and assuming the statistical properties of the system noise and measurement noise are known ("white" noise with zero mean and normal probability density function (pdf), the usual Kalman filter algorithms, given in eqs (13-17), can be used to esti- mate the slope, the intercept, and the drift parameters of the calibration curve [7]

$$
\begin{aligned}
& \mathbf{x}(k / k-1)=\mathbf{F}(k) \hat{\mathbf{x}}(k-1 / k-1) \\
& \mathbf{P}(k / k-1)=\mathbf{F}(k) \mathbf{P}(k-1 / k-1) \mathbf{F}^{\prime}(k)+Q(k-1) \\
& \mathbf{x}(k / k)=\hat{\mathbf{x}}(k / k-1)+\mathbf{k}(k)\left\{z(k)-\mathbf{h}^{\prime}(k) \hat{\mathbf{x}}(k / k-1)\right. \\
& \mathbf{P}(k / k)=\mathbf{P}(k / k-1)-\mathbf{k}(k) \mathbf{h}^{\prime}(k) \mathbf{P}(k / k-1) \\
& \mathbf{k}(k)=\mathbf{P}(k / k-1) \mathbf{h}(k)\left\{\mathbf{h}^{\prime}(k) \mathbf{P}(k / k-1) \mathbf{h}(k)+\right. \\
& \quad R(k)\}^{-1}
\end{aligned}
$$

where:

$Q(k):$ system noise

$\boldsymbol{R}(k)$ : measurement noise

$\mathbf{k}(k)$ : Kalman gain factor (correction factor)

Equation (18) gives the innovation, i.e., the difference between the experimental and the estimated measurement

$$
\nu(k)=z(k)-\mathbf{h}^{t}(k) \hat{\mathbf{x}}(k / k-1)
$$

with

$E\{v(k)\}=0$

$E\left\{v(k) v^{t}(l\}=\left\{\mathbf{h}^{t}(k) \mathbf{P}(k / k-1) \mathbf{h}(k)+R(k)\right\} \delta(k, l)\right.$.

The variance of the predicted measurement is also 
given by eq (19). The prediction of the Kalman filter can be used to evaluate a constituent in an unknown sample. Rewriting the calibration relation eq (3) gives

$$
\begin{gathered}
\hat{c}_{\mathrm{un}}=(z(k)-b) / \hat{\mathbf{a}} \\
\operatorname{var}\left(\hat{c}_{\mathrm{un}}\right)=\left(1 / \hat{a}^{2}\right)\left\{\mathbf{h}_{u n}^{f_{u n}} \mathbf{P}(k / k-1) \mathbf{h}_{\mathrm{un}}+\mathbf{R}_{\mathrm{un}}\right.
\end{gathered}
$$

with: $\quad \boldsymbol{h}_{u n}^{\prime}=\left(\hat{c}_{\mathrm{um}}, 1,0,0\right)$ and $\hat{a}, \hat{b}$ from $\hat{\boldsymbol{x}}(k / k-1)$.

The relative imprecision of an unknown concentration $c_{\mathrm{un}}$ is given by

$$
N_{\mathrm{un}}=2 /\left(c_{\mathrm{un}} \hat{a}\right)\left\{\mathbf{h}_{\mathrm{un}}^{c} \mathbf{P}(k / k-1) \mathrm{h}_{\mathrm{un}}+R_{\mathrm{un}}\right\}^{\frac{1}{2}}
$$

with: $\mathbf{h}_{\text {un }}^{t}=\left(c_{\text {un }}, 1,0,0\right)$.

The available calibration standards are used to compute $N_{u n}$ in eq (21). The computed maximum imprecision $N_{\max }$ is compared with a predefined imprecision $N_{\text {crit. }}$ If $N_{\max } \geqslant N_{\text {cril, }}$ a recalibration is performed; if $N_{\max }<N_{\text {crit }}$ the samples are processed.

\section{Application in Practice}

The described state estimation is applied in automated flow injection analysis. The quality of the results is improved by smoothing all the stored estimates of the Kalman filter. An extensive description of the smoothing procedure is given in [8].
Figure 2 shows an automated flow injection system used for the determination of chloride in aqueous sam. ples. Thiocyanate originating from $\mathrm{Hg}(\mathrm{SCN})_{2}$ is substituted by $\mathrm{Cl}^{-}$in the presence of $\mathrm{Fe}^{3+}$. Red coloured $\mathrm{Fe}(\mathrm{SCN})_{3}$ is formed and measured spectrophotometrically at $470 \mathrm{~nm}$. Each sample requires 40 seconds; 90 samples/hour can be processed.

Figure 3 shows the results of the repeated injections of $2,4,6,8$, and $10 \mathrm{ppm}$ samples. The chi-square values given in table 1 are obtained with a noise variance $R=15.10^{-6}$ and system noise covariances $Q_{33}=Q_{44}=10^{-10}$.

As can be seen from table 1, a first order calibration graph (4 parameter state, $x 1-x 4$ ) is obviously not satisfying and the model has to be extended to a second order drifting calibration graph (6 parameter state, $x \mathrm{l}-x 6$ ). In this case smoothing does not yield significant improvement in the estimation.

Figure 4 shows the result of the measurement of standards and "unknown" samples (6 ppm) at fixed positions in the sequence. The on-line Kalman estimation is depicted in figure 5 and the improvement by the smoother is shown in figure 6 . The histograms figure 7 and figure 8 show the evaluated results.

The on-line processing of the uncorrected peak heights permits one to decide to recalibrate or not. Figure $9 \mathrm{a}$ and figure 10 give an impression of the results, respectively for the estimation of the state by the Kalman filter and after the smoothing. If a given preset criterian $N_{\text {crit }}$ is exceeded by the maximum imprecision $N_{\text {max }}$ the system is recalibrated. After 9-12 calibrations the system starts to evaluate the unknown samples and recalibrates regularly.

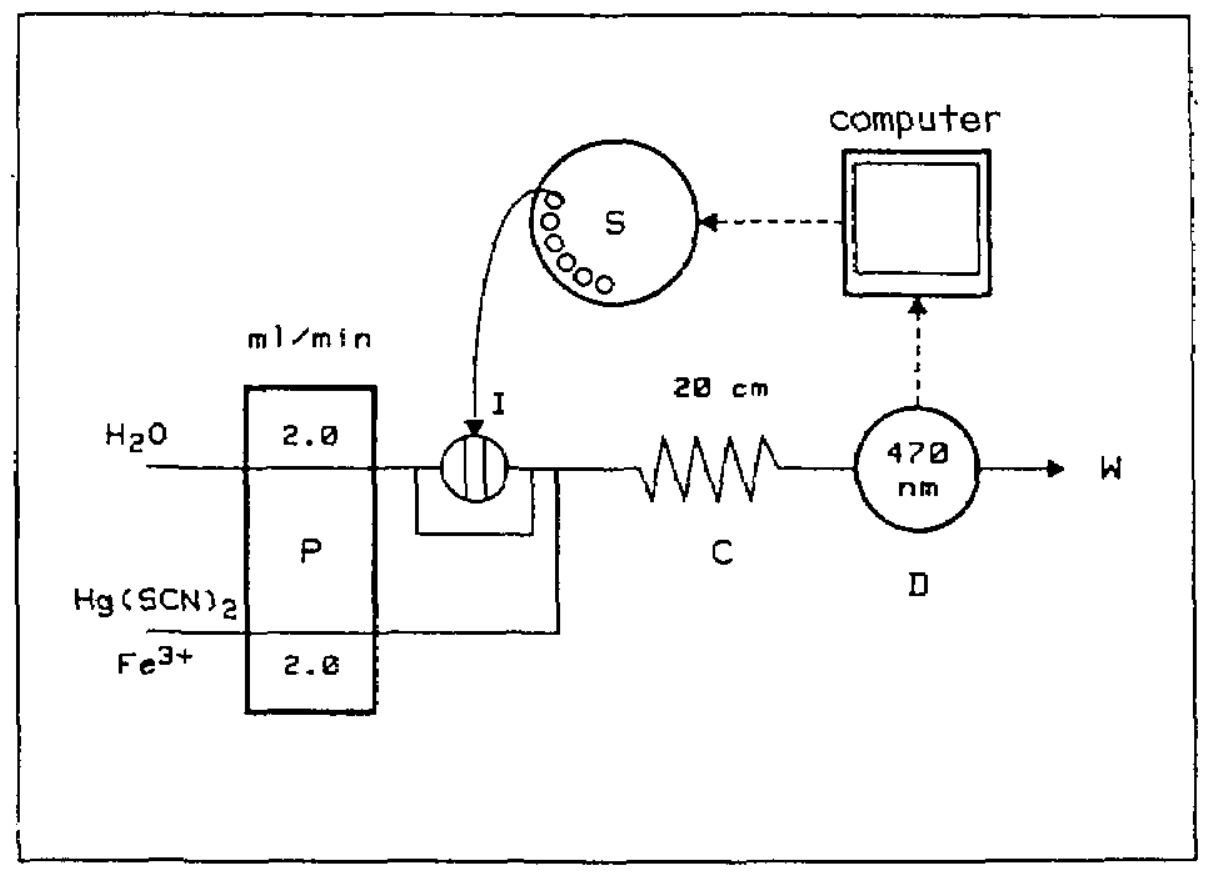

Figure 2-Flow injection systern. $S=$ sample holder, $C=$ column, $\mathrm{P}=$ pump, $\mathrm{D}=$ spectrophotometer, $I=$ injection valve, $W=$ waste. 


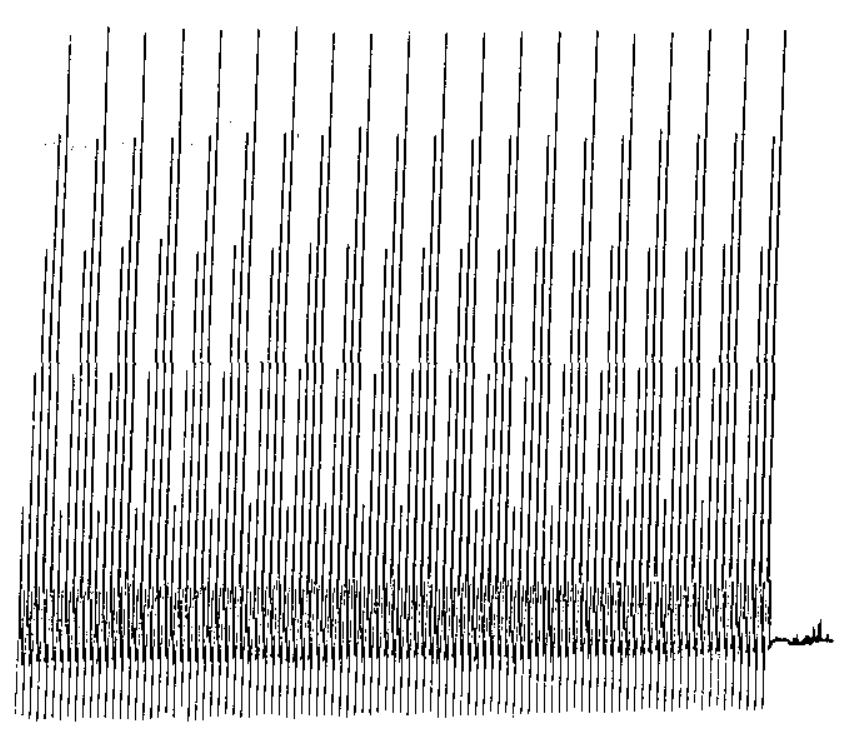

Figure 3-Repeated injections.

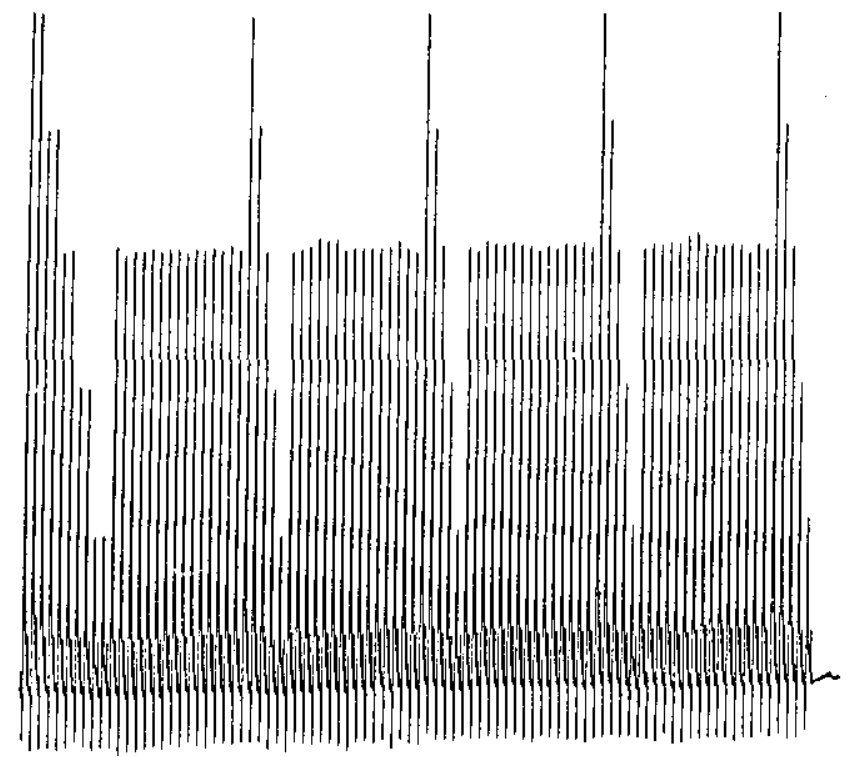

Figure 4-Standards and unknown samples.

Table 1. Values of $x^{2}$ for the flow injection peaks of figure 3.

\begin{tabular}{lcccc}
\hline \hline & Peak height & $\begin{array}{c}\text { Peak height } \\
\text { baseline corrected }\end{array}$ & Peak integral/300 & $\begin{array}{c}\text { Peak integral/300 } \\
\text { corrected }\end{array}$ \\
\hline $\begin{array}{l}\text { Kalman filter } \\
\text { (1st order) }\end{array}$ & 618.8 & 662.7 & 134.9 & 797.2 \\
$\begin{array}{l}\text { Smoothed } \\
\text { (1st Order) }\end{array}$ & 578.7 & 620.1 & 122.0 & 744.2 \\
$\begin{array}{l}\text { Kalman filter } \\
\text { (2nd order) }\end{array}$ & 69.8 & 67.5 & 61.0 & 395.4 \\
$\begin{array}{l}\text { Smoothed } \\
\text { (2nd order) }\end{array}$ & 62.0 & 59.3 & 53.8 & 354.4 \\
\hline
\end{tabular}

Figure 5-On-line Kalman estimation.

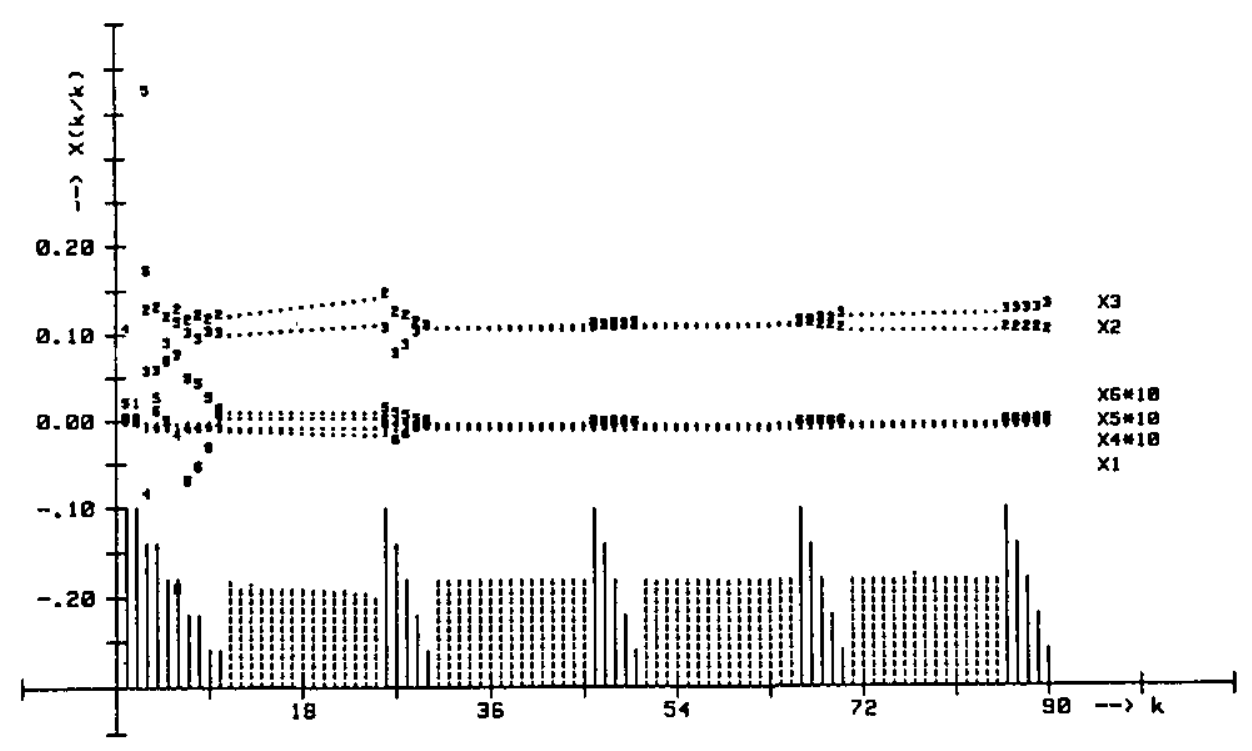




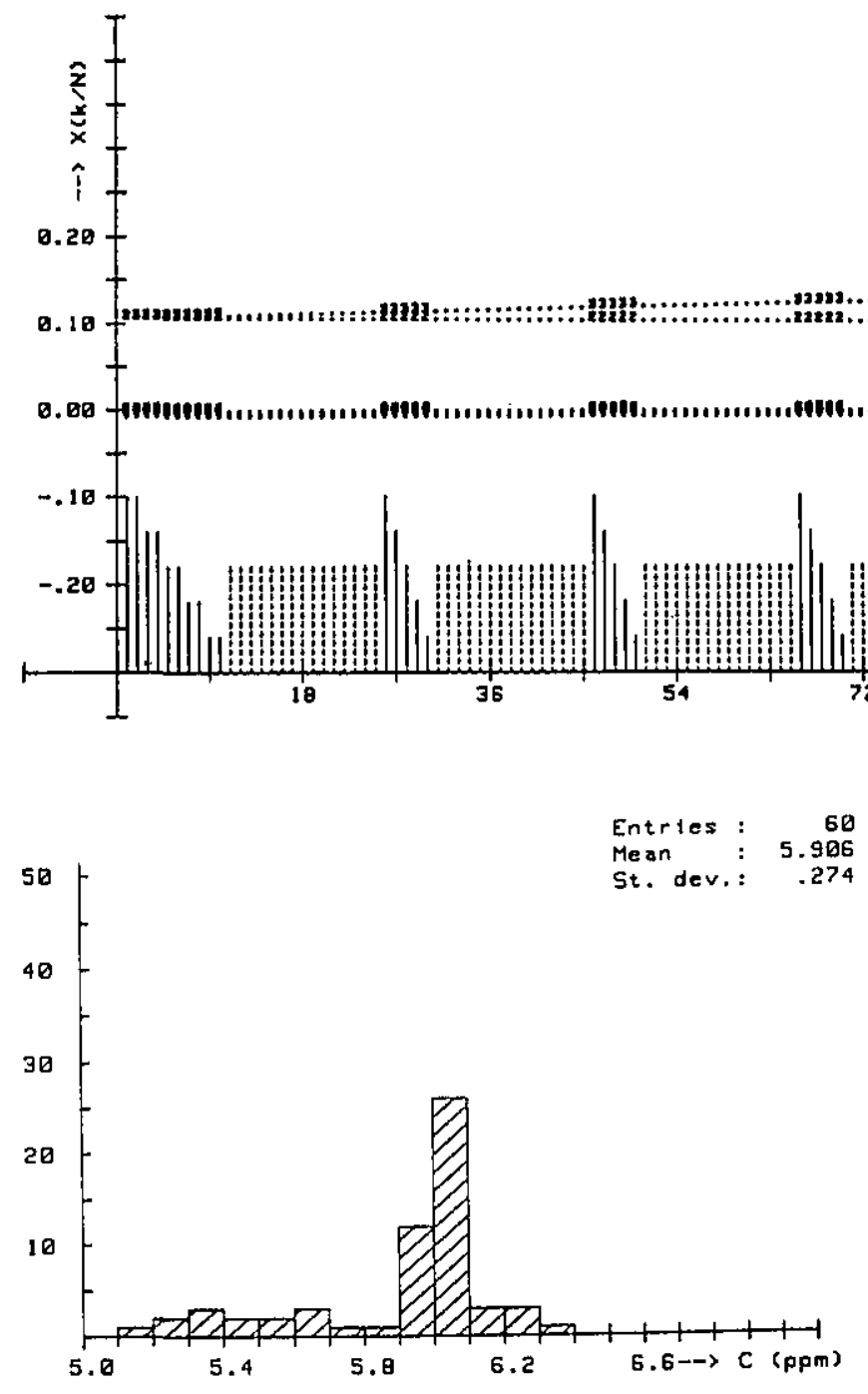

Figure 7-Histogram of the Kalman estimation.
Figure 6-Improvement by smoothing.

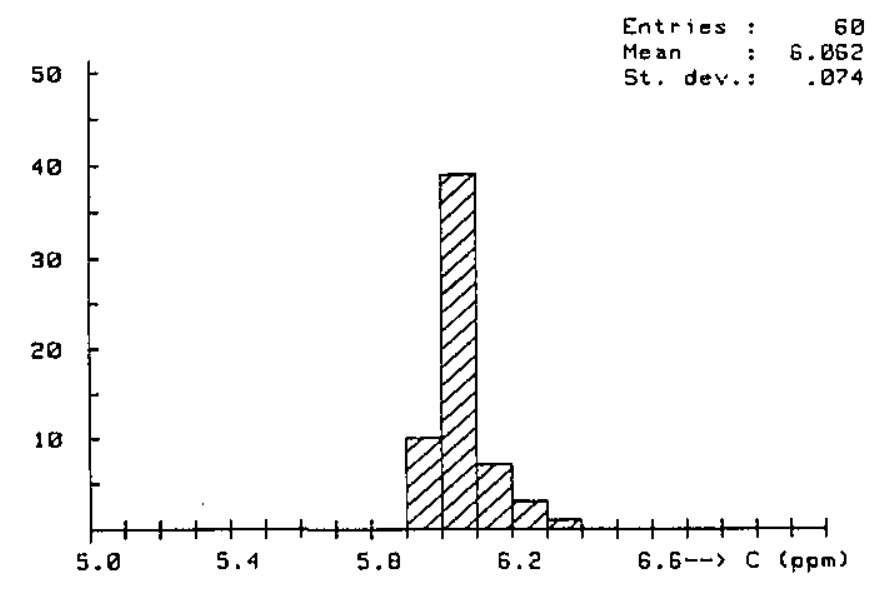

Figure 8-Histogram of the smoothed results.

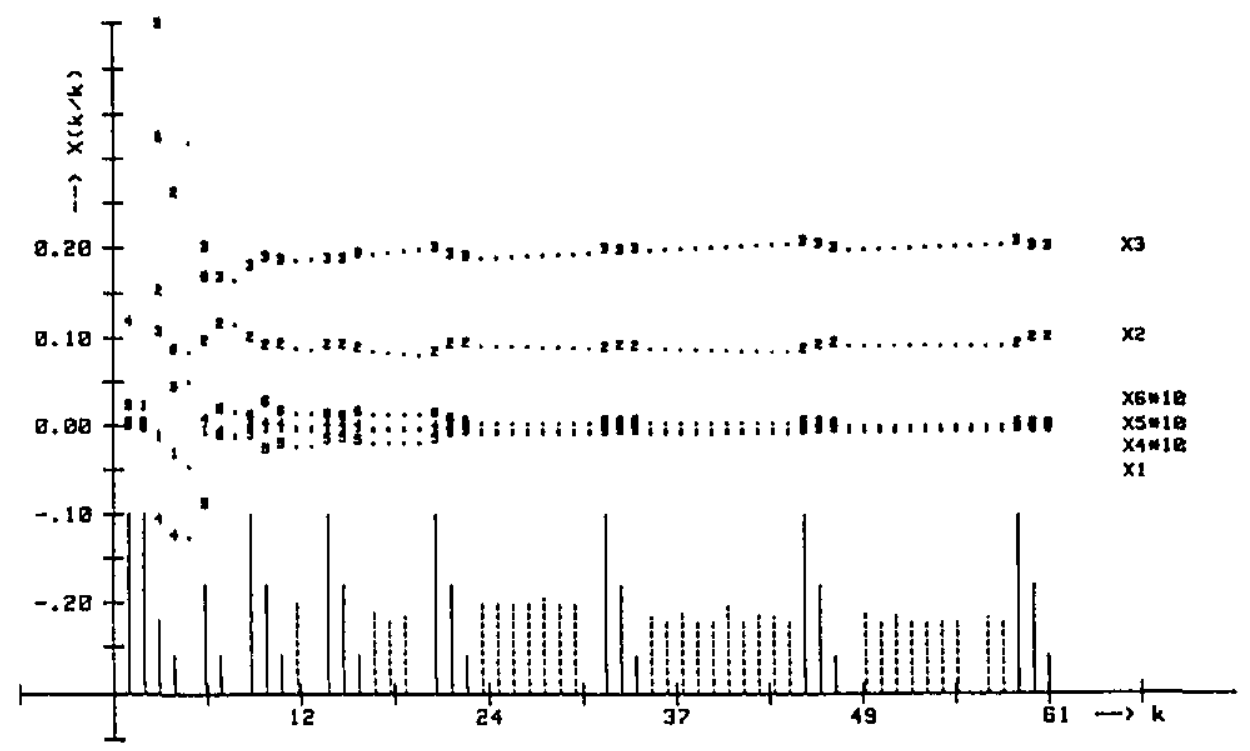

Figure 9-On-line calibration system. State and concentrations used by the Kalman filter. 
Figure 10-On-line calibration system. State and concentrations used by the smoother.

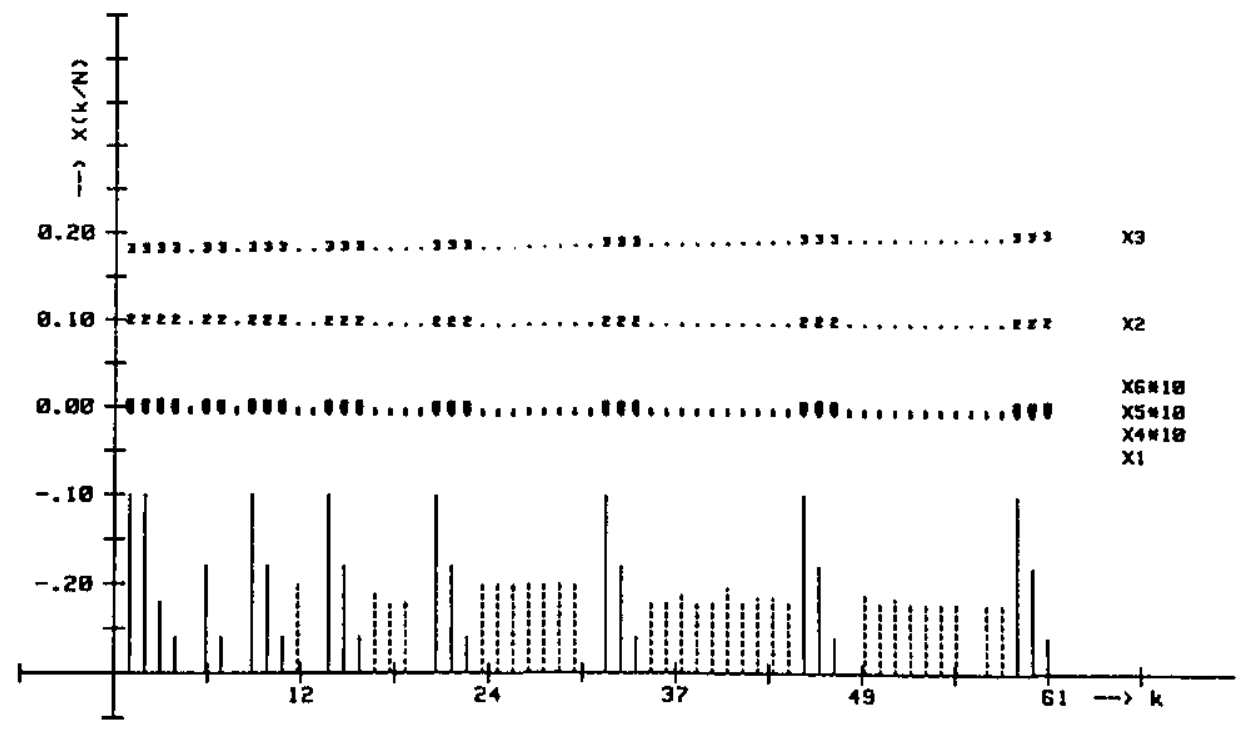

However, the autocorrelation function of $x(t)$ is defined as:

$$
R_{x x}\left(t_{1}, t_{2}\right)=E\left[x\left(t_{1}\right), x\left(t_{2}\right)\right]
$$

and eqs (25) and (26) can be combined to:

$$
R_{x y}\left(t_{1}, t_{2}\right)=\int_{0}^{\infty} h(\tau) R_{x x}\left(t_{1}, t_{2}-\tau\right) d \tau(\tau>0)
$$

If $x(t)$ is stationary, then:

$$
R_{x x}\left(t_{1}, t_{2}-\tau\right)=R\left(t_{2}-\tau-t_{1}\right)=R(t-\tau)
$$

and eq (27) becomes:

$$
R_{x y}\left(t_{1}, t_{2}\right)=R_{x y}(t)=\int_{0}^{\infty} h(\tau) R_{x x}(t-\tau) d \tau
$$

Comparing eq (23) and eq (29) shows that the output signal $y(t)$ of a linear system with an input signal equal to the autocorrelation function $R_{x x}(t)$ of a signal $x(t)$ is similar to the cross-correlation function $R_{x y}(t)$ of the input signal $x(t)$ and the output signal $y(t)$ resulting from $x(t)$.

A white noise, that is, white with respect to the bandwidth of the system, has in impulse-shaped autocorrelation function and can be used as input function $x(t)$ to determine the impulse response, in which case $R_{\mathrm{xy}}(t)=h(t)$.

On further consideration a chromatographic procedure can be regarded as the determination of an impulse response; a chromatogram shows the response on the impulse-shaped injection of the sample. The prime objective of correlation chromatography is to determine the chromatogram by stochastically injecting the sam- 
ple into the column and cross-correlating the input and the resulting output. If the chromatographic system is contaminated with noise, this noise is not correlated with the input and its contribution to the overall crosscorrelation function converges to zero with increasing correlation time. A considerable improvement of the signal to noise ratio can be achieved in a relatively short time.

\section{Application in Practice}

The most suitable random input function, controlling the input flow of the sample, is the pseudo random binary sequence (PRBS). This function is to be preferred to other random inputs with approximately impulseshaped autocorrelation functions for the following reasons:

1) It is a binary noise, the only two levels being +1 and -1 or +1 and 0 . The levels can be used to control simple on/off valves and correspond with the injection of sample and eluent, respectively;

2) It can be easily generated and reproduced; and,

3) Its special properties offer the possibility of reducing the correlation noise, which is caused by a limited correlation time.

The PRBS is a logical function combining the properties of a true binary random signal with those of a reproducible deterministic signal. After a certain time (a sequence) the pattern is repeated. It is important that the estimated autocorrelation function of a PRBS, if computed over an integral number of sequences, is at all times exactly equal to the autocorrelation function. Fig. ure 11 shows a correlation HPLC set-up.

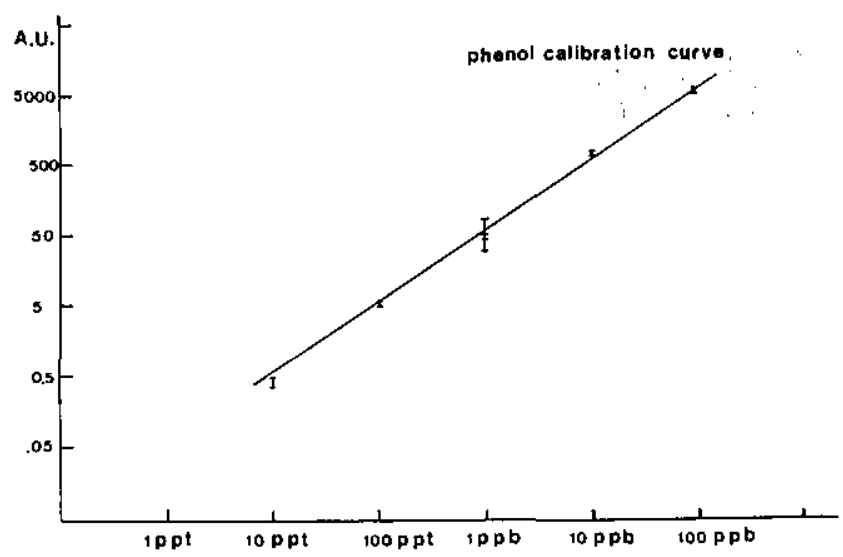

Figure 12-Calibration graph of phenol with fluorimetric detection.

The analytical performance of $\mathrm{CC}$ is demonstrated in figure 12. A calibration curve of phenol was measured over five decades of concentration: $0.01-100 \mu \mathrm{g} l^{-1}$. Conventional HPLC equipment with fluorimetric detection and a newly developed injection device for correlation HPLC was used. The two higher concentrations $\left(10-100 \mu \mathrm{g} l^{-1}\right)$ were determined by conventional (reverse phase) HPLC and the two lower concentrations $\left(0.01-0.1 \mu \mathrm{gl}^{-1}\right)$ by correlation HPLC with 16 and 3 sequences of correlation time, respectively.

Measurements at the $1 \mu \mathrm{g}^{-1}$ level were performed both by conventional and correlation HPLC (1 sequence).

The bars indicated on the calibration graph represent the peak area $\pm 3 \sigma_{1}$ (arbitrary units), when $\sigma_{1}$ is the standard deviation of the integrated noise [10]. The inner bars at the $1 \mu \mathrm{g}$ level represent the correlation re-

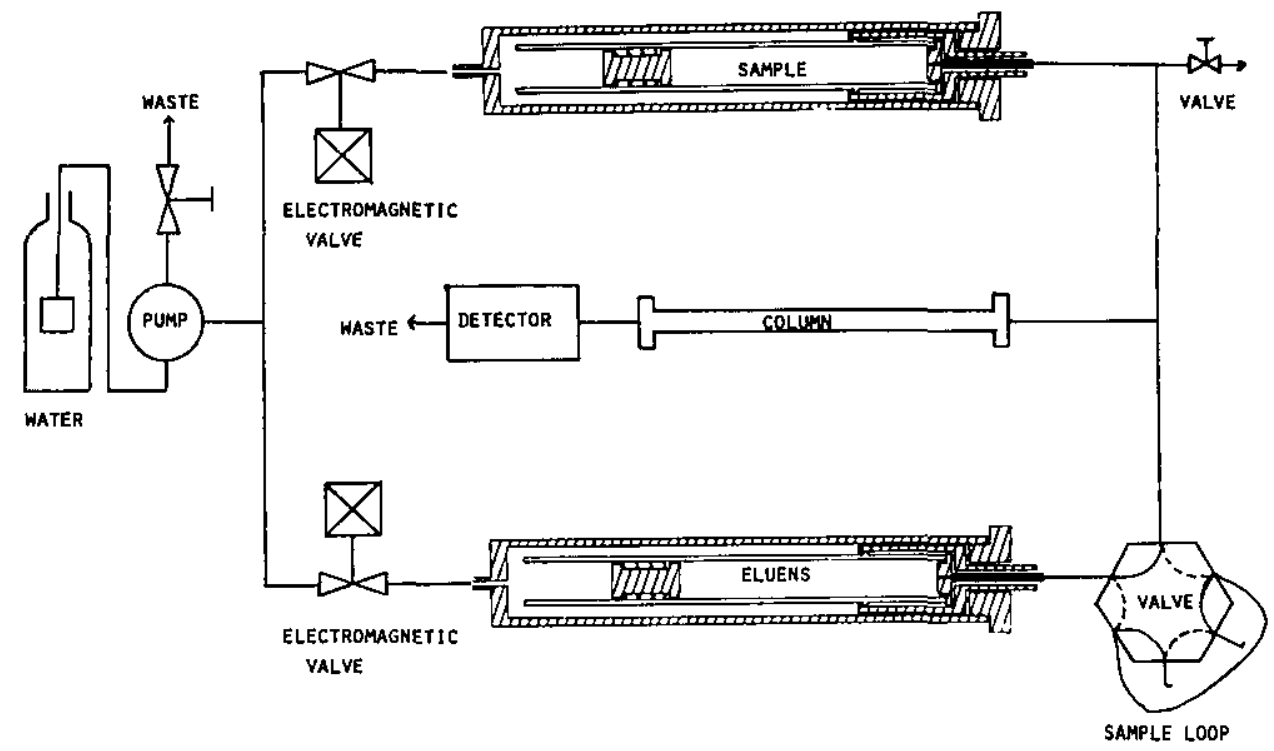

Figure 11-Set-up of a correlation HPLC system. The constant water flow is depending on a PRBS pattern directed either to the sample or to the eluent reservoir. 
sults and the outer bars the single injection results. The detection limit for the single injection experiments, defined as $3 \sigma_{1}$, was about $0.5 \mu \mathrm{g} l^{-1}$. The detection limit with the $10 \mathrm{ng} l^{-1}$ concentration was estimated to be 3 $\mathrm{ng} l^{-1}(3 \mathrm{ppt})$.

\section{Simultaneous Correlation Chromatography}

On further consideration, the question arises whether it is possible to inject different samples simultaneously, each sample injection controlled by its own unique PRBS. If these pseudo random sequences are mutually uncorrelated, then the correlogram (chromatogram) of each sample can be determined without any influence of the other samples, even if the components in the samples are the same. The problem is to find uncorrelated sequences.

A possible solution is the following. A binary pseudo random noise sequence is generated by a digital shift register with a suitable modulo-2 feedback. Appropriate digital multiplexing yields multiple uncorrelated pseudo random outputs from a single $n$-bit shift register. Each of the $K$ output sequences is identical to the single shift register sequence; they are staggered by $2^{n} / K$ bits, so they will remain uncorrelated for $1 / K\left(2^{n}-1\right)$ output bits. These output sequences can be used for the mentioned simultaneous input patterns of a chromatographic column.

Figure 13 shows the result of a simulation experiment. A separation of two components is simulated with four different samples which were "injected" simultaneously. The "separation" is excellent.

The injection of $n$ samples requires a correlation time of $n$ times the total elution time. Therefore, no time gain can be expected. However, the method can be used for a number of interesting applications. For example, simultaneous $\mathrm{CC}$ permits measurement and calibration at

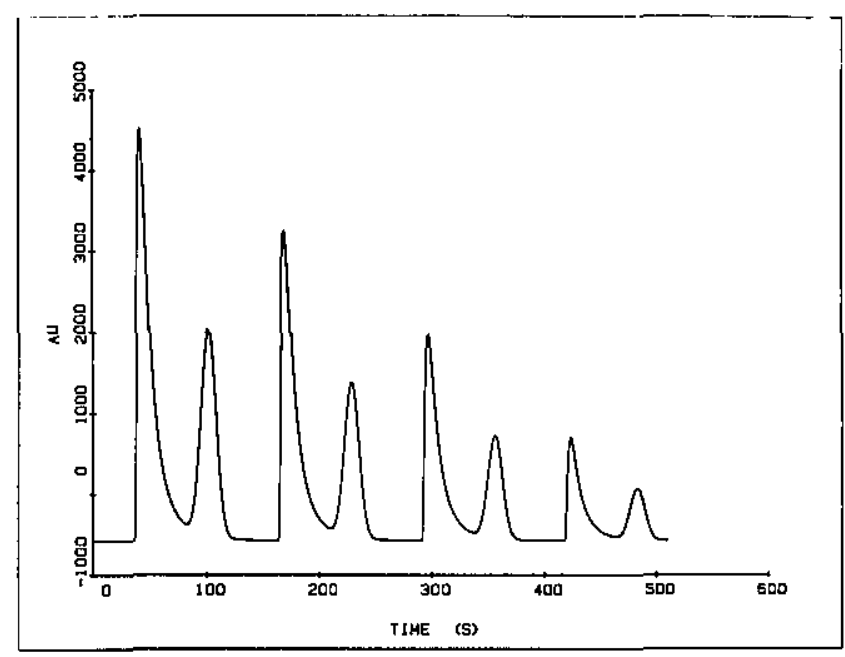

Figure 13-Simulated simultaneous chromatogram.

the same time under the same chromatographic conditions.

Figure 14 shows an experimental set-up of a simultaneous chromatograph (HPLC) with four reservoirs (three samples and eluent) together with four valves, each controlled by a sequence uncorrelated with the others. The flow stability is maintained by dividing a clock period in four parts. In each part either eluent or sample (respectively sample 1,2 , and 3 ) can be injected, depending on the status ( 0 or 1$)$ of the sequence concerned.

The results of the analysis of three samples with naphtalene, anthracene, and 1,2 benzanthracene is shown in figure 15. The anthracene concentration in each sample is the same; the concentration of the other components in the different samples differ by a factor of 2 .

The experimental injection system is not yet perfect, and because of this, serves as a source of so-called correlation "noise." This noise is not really random, but is

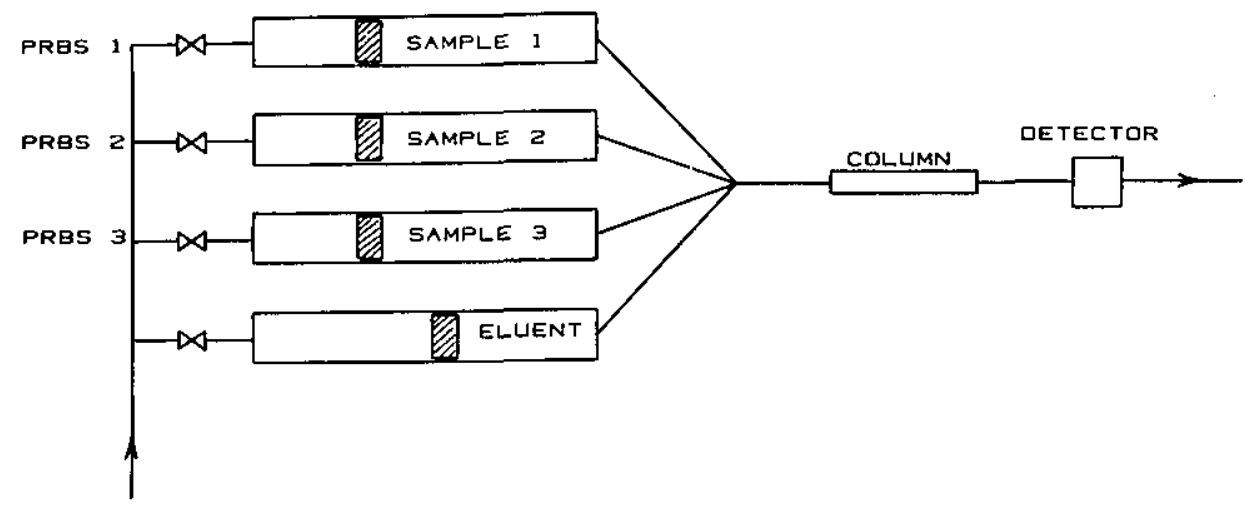

Figure 14-Experimental set-up of a simultaneous HPLC system. 


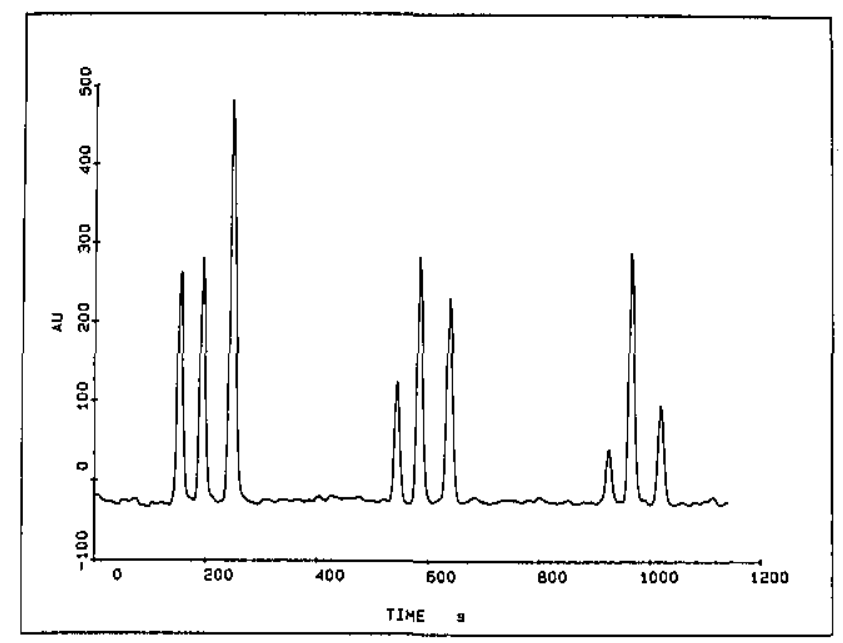

Figure 15-Simultaneous chromatogram of three samples, each containing naphtalene, anthracene and 1,2 benzanthracene with different concentrations.

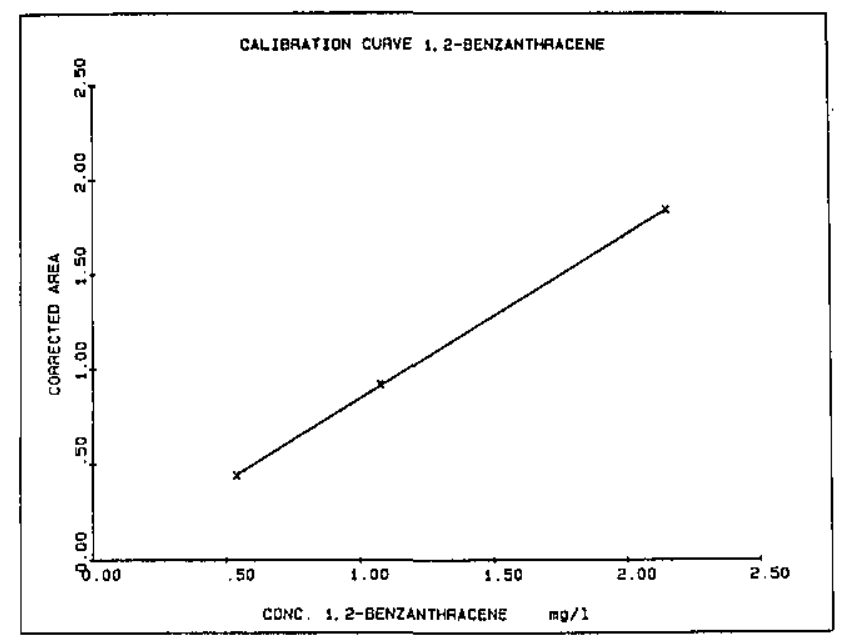

Figure 16-Calibration graph for benzanthracene, simultaneously determined.

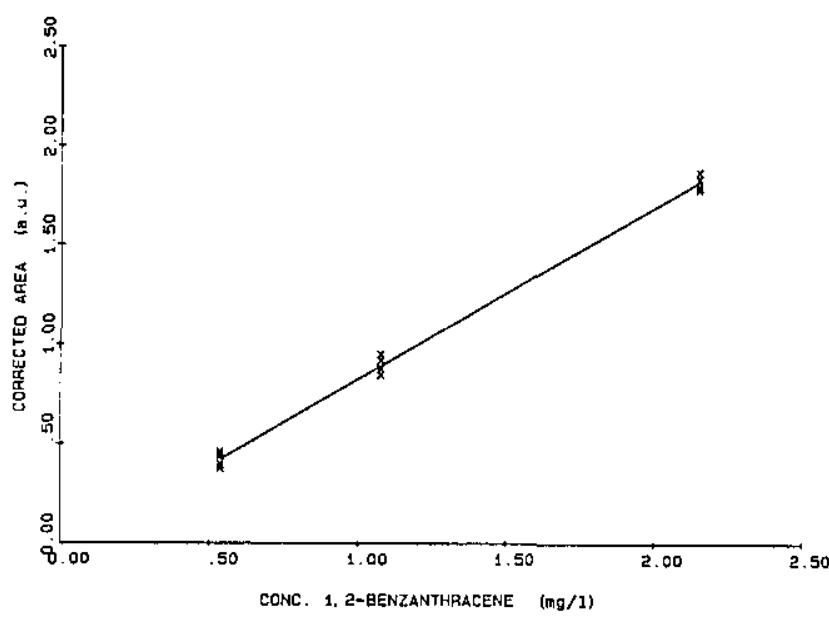

Figure 17-Calibration graph for benzanthracene, successively determined.

composed of deterministic signals (ghost peaks). The peaks of benzanthracene are used to construct a calibration curve (fig. 16). Comparison of this curve with the calibration curve determined in the usual way (fig. 17) shows the performance of the method. The advantages are twofold: the random fluctuations are reduced by the multiple injection and averaging property, and both the unknown sample and the calibration sample are measured simultaneously under exactly the same conditions.

The final conclusion is that on-line chemometric techniques, such as Kalman filtering and correlation procedures, create promising new possibilities in analytical chemistry. The given improvements of the calibration procedure are a typical example of the power of these techniques.

Major contributions to this paper were made by P. C. Thijssen, J. M. Laeven and C. Mars.

\section{References}

[1] Thijssen, P. C.; S. M. Wolfrum, G. Kateman, and H. C. Smit, Anal. Chim. Acta 256, 87 (1984).

[2] Nisbet, J. A., and E. Simpson, Clin. Chim. Acta 39, 367 (1972).

[3] Nisbet, J. A., and J. A. Owen, Clin. Chim. Acta 92, 367 (1979).

[4] Bennett, A.; D. Gartelman, J. J. Mason, and J. A. Owen, Clin. Chim. Acta 29, 161 (1970).
[5] Svehla, G., and E. L. Dickson, Anal. Chim. Acta 136, 369 (1982).

[6] Mclelland, A. S., and A. Fleck, Ann. Clin. Biochem. 15, 281 (1978).

[7] Gelb, A., (Ed.), Applied Optimal Estimation, MIT Press, Cambridge, MA, USA (1974).

[8] Thijssen, P. C.; G. Kateman and H. C. Smit, Anal. Chim. Acta (submitted for publication, 1985).

[9] Smit, H. C.; T. T. Lub and W. J. Vloon, Anal. Chim. Acta 122, 267 (1980).

[10] Smit, H. C., and H. L. Walg, Chromatographia 8, 311 (1975). 